\title{
Subdiffusive master equation with space-dependent anomalous exponent and structural instability
}

DOI:

10.1103/PhysRevE.85.031132

\section{Document Version}

Final published version

Link to publication record in Manchester Research Explorer

\section{Citation for published version (APA):}

Fedotov, S., \& Falconer, S. (2012). Subdiffusive master equation with space-dependent anomalous exponent and structural instability. Physical Review E - Statistical, Nonlinear, and Soft Matter Physics, 85(3), [031132]. https://doi.org/10.1103/PhysRevE.85.031132

\section{Published in:}

Physical Review E - Statistical, Nonlinear, and Soft Matter Physics

\section{Citing this paper}

Please note that where the full-text provided on Manchester Research Explorer is the Author Accepted Manuscript or Proof version this may differ from the final Published version. If citing, it is advised that you check and use the publisher's definitive version.

\section{General rights}

Copyright and moral rights for the publications made accessible in the Research Explorer are retained by the authors and/or other copyright owners and it is a condition of accessing publications that users recognise and abide by the legal requirements associated with these rights.

\section{Takedown policy}

If you believe that this document breaches copyright please refer to the University of Manchester's Takedown Procedures [http://man.ac.uk/04Y6Bo] or contact uml.scholarlycommunications@manchester.ac.uk providing relevant details, so we can investigate your claim.

\section{OPEN ACCESS}




\title{
Subdiffusive master equation with space-dependent anomalous exponent and structural instability
}

\author{
Sergei Fedotov and Steven Falconer \\ School of Mathematics, The University of Manchester, Manchester M60 1QD, United Kingdom
}

(Received 11 December 2011; published 21 March 2012)

\begin{abstract}
We derive the fractional master equation with space-dependent anomalous exponent. We analyze the asymptotic behavior of the corresponding lattice model both analytically and by Monte Carlo simulation. We show that the subdiffusive fractional equations with constant anomalous exponent $\mu$ in a bounded domain $[0, L]$ are not structurally stable with respect to the nonhomogeneous variations of parameter $\mu$. In particular, the GibbsBoltzmann distribution is no longer the stationary solution of the fractional Fokker-Planck equation whatever the space variation of the exponent might be. We analyze the random distribution of $\mu$ in space and find that in the long-time limit, the probability distribution is highly intermediate in space and the behavior is completely dominated by very unlikely events. We show that subdiffusive fractional equations with the nonuniform random distribution of anomalous exponent is an illustration of a "Black Swan," the low probability event of the small value of the anomalous exponent that completely dominates the long-time behavior of subdiffusive systems.
\end{abstract}

DOI: 10.1103/PhysRevE.85.031132

PACS number(s): 05.40.Fb, 02.50.-r, 05.10.Gg

\section{INTRODUCTION}

The last decade has seen increasingly detailed development of the fractional equations describing the anomalous transport in physics, biology, and chemistry [1-4]. Special attention has been paid to slow subdiffusive transport for which meansquared displacement is sublinear $\left\langle x^{2}(t)\right\rangle \sim t^{\mu}$, where $\mu$ is the anomalous exponent $\mu<1$. Subdiffusion is experimentally observed for proteins and lipids on cell membranes [5], RNA molecules in the cells [6], transport in spiny dendrites [7], etc. The major feature of this process is the absence of the characteristic microscopic time scale. The theory of anomalous subdiffusion leads to fractional partial differential equations involving memory effects. If we introduce the probability density function $p(x, t)$ for finding the particle in the interval $(x, x+d x)$ at time $t$, then the subdiffusive transport of the particles under the influence of external time-independent force can be described by the fractional Fokker-Planck (FFP) equation,

$$
\frac{\partial p}{\partial t}=\mathcal{D}_{t}^{1-\mu} L_{F P} p
$$

with

$$
L_{F P} p=-\frac{\partial\left(v_{\mu}(x) p\right)}{\partial x}+\frac{\partial^{2}\left(D_{\mu}(x) p\right)}{\partial x^{2}} ;
$$

(see excellent reviews [1] and [2]). The Riemann-Liouville derivative $\mathcal{D}_{t}^{1-\mu}$ is defined as

$$
\mathcal{D}_{t}^{1-\mu} p(x, t)=\frac{1}{\Gamma(\mu)} \frac{\partial}{\partial t} \int_{0}^{t} \frac{p(x, u) d u}{(t-u)^{1-\mu}},
$$

and the anomalous exponent $\mu<1$ is assumed to be constant.

The central result of this paper is that the subdiffusive fractional equations with constant $\mu$ in a bounded domain $[0, L]$ are not structurally stable with respect to the nonhomogeneous variations of parameter $\mu$. It turns out that the space variations of the anomalous exponent lead to a drastic change in asymptotic behavior of $p(x, t)$ for large $t$. To show this high sensitivity to nonhomogeneous perturbations, one can consider the following exponent:

$$
\mu(x)=\mu+\delta v(x)
$$

with constant $\mu$ and perturbation $\delta v(x)$ (see Fig. 1). The asymptotic long-time behavior of the density $p(x, t)$ with (4) is quite different from that of the solution to Eq. (1) with the constant value of $\mu$. It means that the standard subdiffusive equation with constant $\mu$ is not a robust model for subdiffusive transport in heterogeneous complex media.

Now let us explain our main result. The standard way to deal with the fractional equation like (1) in the bounded domain $[0, L]$ is a method of separation of variables [1]. Let us consider the case of the reflecting boundaries at $x=0$ and $x=L$ when (1) has a stationary solution $p_{s t}(x)$ satisfying

$$
v_{\mu}(x) p_{s t}=\frac{\partial}{\partial x}\left(D_{\mu}(x) p_{s t}\right) .
$$

We can write a partial solution of Eq. (1) in the form,

$$
p(x, t)=p_{s t}(x) Q(x) T(t) .
$$

The time evolution $T(t)$ is described by the fractional relaxation equation,

$$
\frac{\partial T}{\partial t}=-\lambda \mathcal{D}_{t}^{1-\mu} T
$$

where $\lambda$ is the separation constant. The function $Q(x)$ satisfies

$$
L_{F P}^{*} Q=-\lambda Q \text {. }
$$

Here the operator $L_{F P}^{*}$ is the adjoint to $L_{F P}$

$$
L_{F P}^{*} Q=v_{\mu}(x) \frac{\partial Q}{\partial x}+D_{\mu}(x) \frac{\partial^{2} Q}{\partial x^{2}} .
$$

Thus the solution of Eq. (1) can be written as

$$
p(x, t)=p_{s t}(x) \sum_{n=0}^{\infty} E_{\mu}\left(-\lambda_{n} t^{\mu}\right) Q_{n}(x) p_{0 n},
$$

where $p_{0 n}=\int_{0}^{L} p_{0}(x) Q_{n}(x) d x$ and $Q_{n}(x)$ are the eigenfunctions of $(7)\left(Q_{0}(x)=1\right)$. The details can be found in a book [8] on page 129 (see also [9] for a fractional case). The essential difference between the standard Fokker-Planck equation and the FFP equation is the rate of relaxation of $p(x, t) \rightarrow p_{s t}(x)$. In the anomalous subdiffusive case the relaxation process is very slow and it is described by a Mittag-Leffler function 


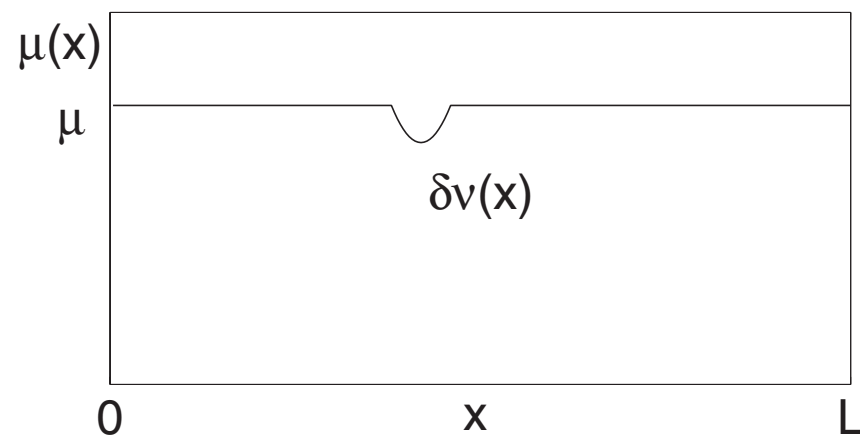

FIG. 1. Nonuniform distribution of anomalous exponent $\mu(x)$ on the interval $[0, L]$.

$E_{\mu}\left(-\lambda_{n} t^{\mu}\right)$ with the power-law decay $t^{-\mu}$ as $t \rightarrow \infty$ [1-3] (see also [10]). The exponential decay $\exp \left(-\lambda_{n} t\right)$ is recovered for $\mu=1$.

In this paper we show that if we consider nonuniform perturbations of the anomalous exponent as (4), this relaxation picture is completely changed. The method of separation of variables does not work for space-dependent $\mu(x)$. The asymptotic behavior of $p(x, t)$ as $t \rightarrow \infty$ is essentially different from that given by Eq. (9). It turns out that in the limit $t \rightarrow \infty$ the probability density $p(x, t)$ concentrates around the point $x$, where the perturbation $\delta v(x)$ is located, while the stationary distribution $p_{s t}(x)$ is completely irrelevant (see Figs. 2 and 3 ).

\section{FRACTIONAL MASTER EQUATION WITH SPACE-DEPENDENT ANOMALOUS EXPONENT}

The question is how to take into account the nonuniform distribution of the anomalous exponent $\mu$. We cannot simply substitute the expression like (4) into (1). So we need a fractional master equation with space-dependent $\mu(x)$. Chechkin, Gorenflo, and Sokolov were the first to derive the fractional diffusion equation with a varying fractional exponent [11]. They studied a composite system with only two separate regions with different anomalous exponents and found interesting effects involving a nontrivial average drift. A similar phenomenon has been analyzed in terms of two equations with a different exponent by Korabel and Barkai [12]. Anomalous diffusion in composite media with spacedependent exponent $\mu$ has been also considered in Ref. [13].

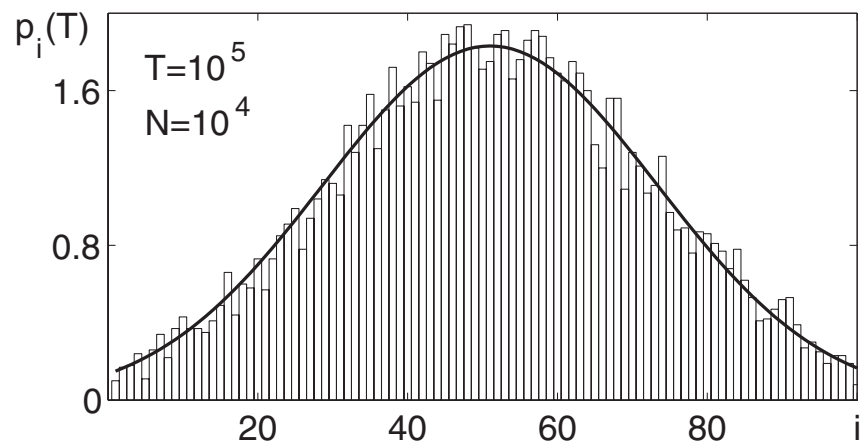

FIG. 2. Long-time limit of the solution to the system (41) with $\mu_{i}=0.5$ for all $i$. Gibbs-Boltzmann distribution is represented by the line.

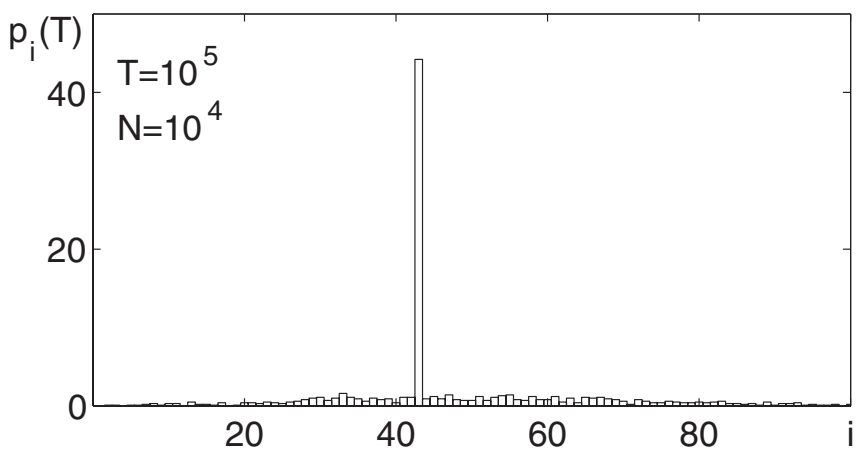

FIG. 3. Long-time limit of the system (41) when $\mu_{i}$ is subject to a perturbation. The parameters are $\mu_{i}=0.5$ for all $i$ except $i=42$ for which $\mu_{42}=0.3$.

\section{A. Hazard function and structured probability density function}

Here we present an alternative derivation which is valid for a general space- and time-dependent jump densities. Consider a "space-jump" random walk model in one space dimension. The particle movement can be described as follows. It waits for a random time (residence time) $T_{x}$ at each point $x$ in space before making a jump to another point. The index $x$ indicates that the waiting time $T_{x}$ depends on a space coordinate $x$. It is convenient to define the hazard function [14] as the escape rate of a walker from the point $x$,

$$
\gamma(x, \tau)=\lim _{h \rightarrow 0} \frac{\operatorname{Pr}\left\{\tau<T_{x}<\tau+h\left|T_{x}\right\rangle \tau\right\}}{h} .
$$

The next step is the introduction of the structured probability density function $\xi(x, t, \tau)$ that the particle position $X(t)$ at time $t$ is in the interval $(x, x+d x)$ and its residence time $T_{x}$ at point $x$ is in the interval $(\tau, \tau+d \tau)$. The advantage of the structured density $\xi$ is that a random walk can be considered as Markovian. This is a standard way to deal with non-Markovian processes [14] (see also [15-17]). This density $\xi(x, t, \tau)$ obeys the balance equation,

$$
\frac{\partial \xi}{\partial t}+\frac{\partial \xi}{\partial \tau}=-\gamma(x, \tau) \xi
$$

Here we consider only the case when the residence time of random walker at $t=0$ is equal to zero, so the initial condition is

$$
\xi(x, 0, \tau)=p_{0}(x) \delta(\tau),
$$

where $p_{0}(x)$ is the density for the initial position $X(0)$. The boundary condition at $\tau=0$ can be written as [14]

$$
\xi(x, t, 0)=\int_{\mathbb{R}} \int_{0}^{t} \gamma(x, \tau) \xi(x-z, t, \tau) w(z \mid x-z, t) d \tau d z,
$$

where $w(z \mid x, t)$ is the probability density for jumps $z$ from the point $x$ at time $t$ (jumps are independent from the residence time).

Our purpose now is to derive the fractional master equation for the probability density,

$$
p(x, t)=\int_{0}^{t} \xi(x, t, \tau) d \tau .
$$


It is convenient to introduce the integral escape rate,

$$
i(x, t)=\int_{0}^{t} \gamma(\tau, x) \xi(x, t, \tau) d \tau,
$$

and integral arrival rate,

$$
j(x, t)=\xi(x, t, 0),
$$

as the density of particles with zero residence time. The boundary condition (13) can be rewritten as

$$
j(x, t)=\int_{\mathbb{R}} i(x-z, t) w(z \mid x-z, t) d z .
$$

Differentiation of Eq. (14) with respect to time and substitution of $\partial \xi / \partial t$ from Eq. (11) together with Eq. (17) gives

$$
\frac{\partial p}{\partial t}=\int_{\mathbb{R}} i(x-z, t) w(z \mid x-z, t) d z-i(x, t) .
$$

To close this equation we need to express the escape rate $i(x, t)$ in terms of $p(x, t)$. We solve (11) by the method of characteristics,

$$
\xi(x, t, \tau)=\xi(x, t-\tau, 0) e^{-\int_{0}^{\tau} \gamma(x, s) d s}, \quad \tau<t .
$$

Here we recognize the survival function [14],

$$
\Psi(x, \tau)=\operatorname{Pr}\left\{T_{x}>\tau\right\}=e^{-\int_{0}^{\tau} \gamma(x, s) d s},
$$

so the structural density $\xi$ can be rewritten as

$$
\xi(x, t, \tau)=j(x, t-\tau) \Psi(x, \tau), \quad \tau<t .
$$

The residence time probability density function (PDF) $\phi(x, \tau)$ is related to $\gamma(x, \tau)$ as

$$
\phi(x, \tau)=-\partial \Psi / \partial \tau=\gamma(x, \tau) \exp \left(-\int_{0}^{\tau} \gamma(x, s) d s\right) .
$$

The balance equation for $p(x, t)$ can be found by substitution of Eq. (19) and the initial condition $\xi(x, 0, \tau)=p_{0}(x) \delta(\tau)$ into (14)

$$
p(x, t)=\int_{0}^{t} j(x, u) \Psi(x, t-u) d u+p_{0}(x) \Psi(x, t) .
$$

To obtain the equation for $i(x, t)$ we substitute (19) and the initial condition into (15):

$$
i(x, t)=\int_{0}^{t} j(x, u) \phi(x, t-u) d u+p_{0}(x) \phi(x, t) .
$$

Using the Laplace transform in Eqs. (23) and (24) we eliminate $j(x, t)$ and obtain [11]

$$
i(x, t)=\int_{0}^{t} K(x, t-\tau) p(x, \tau) d \tau,
$$

where $K(x, t)$ is the memory kernel defined by its Laplace transform,

$$
\hat{K}(x, s)=\frac{\hat{\phi}(x, s)}{\hat{\Psi}(x, s)} .
$$

\section{B. Anomalous subdiffusion in heterogeneous media}

Let us consider the anomalous subdiffusive case with the survival probability [18]:

$$
\Psi(x, t)=E_{\mu(x)}\left[-\left(\frac{t}{\tau(x)}\right)^{\mu(x)}\right], \quad 0<\mu(x)<1,
$$

where $E_{\mu}[z]$ is the Mittag-Leffler function. The Laplace transforms of $\Psi(x, t)$ and $\phi(x, t)$ are

$$
\hat{\Psi}(x, s)=\frac{\tau(x)(s \tau(x))^{\mu(x)-1}}{1+(s \tau(x))^{\mu(x)}}, \quad \hat{\phi}(x, s)=\frac{1}{1+(s \tau(x))^{\mu(x)}} .
$$

The Laplace transform of the memory kernel $K(x, t)$ is

$$
\hat{K}(x, s)=\frac{s^{1-\mu(x)}}{\tau(x)^{\mu(x)}},
$$

and the integral escape rate $i(x, t)$ can be written as

$$
i(x, t)=\frac{1}{\tau(x)^{\mu(x)}} \mathcal{D}_{t}^{1-\mu(x)} p(x, t) .
$$

Substitution of this expression into Eq. (18) gives the fractional master equation,

$$
\begin{aligned}
\frac{\partial p}{\partial t}= & \int_{\mathbb{R}} \frac{\mathcal{D}_{t}^{1-\mu(x-z)} p(x-z, t)}{\tau(x-z)^{\mu(x-z)}} w(z \mid x-z, t) d z \\
& -\frac{1}{\tau(x)^{\mu(x)}} \mathcal{D}_{t}^{1-\mu(x)} p(x, t),
\end{aligned}
$$

where $\mathcal{D}_{t}^{1-\mu(x)}$ is the Riemann-Liouville fractional derivative with varying order. This equation can be used to derive the general Fokker-Planck equation [19]. If we assume that the anomalous exponent $\mu$ and time parameter $\tau$ are independent from coordinate $x$, this equation can be rewritten in terms of the Caputo derivative,

$$
\tau^{\mu} \frac{\partial^{\mu} p}{\partial t^{\mu}}=\int_{\mathbb{R}} p(x-z, t) w(z \mid x-z, t) d z-p(x, t) .
$$

It should be noted that the fractional equation with the Caputo derivative cannot be served as a model for subdiffusion in heterogeneous media with varying in the space anomalous exponent $\mu(x)$.

Master equation (31) can be a starting point for deriving nonlinear fractional equations. If instead of $p$ we consider the mean density of particles $\rho$ and assume that jump PDF $w(z)$ depends on $\rho$, then one can write

$$
\begin{aligned}
\frac{\partial \rho}{\partial t}= & \int_{\mathbb{R}} \frac{\mathcal{D}_{t}^{1-\mu(x-z)} \rho(x-z, t)}{\tau(x-z)^{\mu(x-z)}} w(z \mid \rho(x-z, t)) d z \\
& -\frac{1}{\tau(x)^{\mu(x)}} \mathcal{D}_{t}^{1-\mu(x)} \rho(x, t) .
\end{aligned}
$$

Expansion of this equation in $z$ can give a variety of fractional nonlinear partial differential equations. As an example, let us consider the case of the symmetrical kernel $w(z \mid \rho)$ for which the first moment $\int_{\mathbb{R}} z w(z \mid \rho(x, t)) d z=0$. Then (33) can be approximated by a nonlinear fractional equation,

$$
\frac{\partial \rho}{\partial t}=\frac{\partial^{2}}{\partial x^{2}}\left(D_{\mu}(\rho) \mathcal{D}_{t}^{1-\mu(x)} \rho\right),
$$


with varying anomalous exponent $\mu(x)$ and nonlinear fractional diffusion coefficient $D_{\mu}(\rho)$ :

$$
D_{\mu}(\rho)=\frac{m_{2}(\rho)}{2 \tau(x)^{\mu(x)}}, \quad m_{2}(\rho)=\int_{\mathbb{R}} z^{2} w(z \mid \rho) d z .
$$

First, let us consider random walk on a lattice with the space size $a$. We denote the probability of a particle moving right and left from the point $x$ as $r(x)$ and $l(x)$ correspondingly $(r(x)+l(x)=1)$. Then the jump PDF can be written as

$$
w(z \mid x)=r(x) \delta(z-a)+l(x) \delta(z+a) .
$$

The fractional master Eq. (31) takes the form,

$$
\begin{aligned}
\frac{\partial p}{\partial t}= & \frac{r(x-a)}{\tau(x-a)^{\mu(x-a)}} \mathcal{D}_{t}^{1-\mu(x-a)} p(x-a, t) \\
& +\frac{l(x+a)}{\tau(x+a)^{\mu(x+a)}} \mathcal{D}_{t}^{1-\mu(x+a)} p(x+a, t) \\
& -\frac{1}{\tau(x)^{\mu(x)}} \mathcal{D}_{t}^{1-\mu(x)} p(x, t) .
\end{aligned}
$$

In the limit of small $a$ and $\tau(x)$ [20] one can obtain from Eq. (37) the FFP equation with varying the anomalous exponent,

$$
\frac{\partial p}{\partial t}=-\frac{\partial\left(v_{\mu}(x) \mathcal{D}_{t}^{1-\mu(x)} p\right)}{\partial x}+\frac{\partial^{2}\left(D_{\mu}(x) \mathcal{D}_{t}^{1-\mu(x)} p\right)}{\partial x^{2}},
$$

with the finite values of the fractional diffusion coefficient $D_{\mu}(x)$ and fractional drift $v_{\mu}(x)$ :

$$
D_{\mu}(x)=\frac{a^{2}}{2 \tau(x)^{\mu(x)}}, \quad v_{\mu}(x)=\frac{2(r(x)-l(x)) D_{\mu}(x)}{a} .
$$

Note that in order to keep the fractional drift $v_{\mu}(x)$ finite as $a \rightarrow 0$, we need to assume that $r(x)-l(x)=O(a)$.

If we put the reflecting barriers at $x=0$ and $x=L$ and consider constant exponent $\mu$ and diffusion $D_{\mu}$, then the FFP Eq. (38) admits the stationary solution in the form of the GibbsBoltzmann distribution:

$p_{s t}(x)=C \exp [-U(x)], \quad U(x)=-\frac{1}{D_{\mu}} \int^{x} v_{\mu}(z) d z$,

with $C^{-1}=\int_{0}^{L} \exp [-U(x)] d x$.

If $\mu$ is constant, the fractional time derivative does not affect the Gibbs-Boltzmann distribution [1,23]. But this result is structurally unstable with respect to any nonuniform variations of $\mu$. Let us show now that the Gibbs-Boltzmann distribution (40) is absolutely irrelevant for the long-time behavior of the solution to the FFP equation (38) with nonuniform distribution of $\mu(x)$ (4).

\section{Discrete model}

We divide the interval $[0, L]$ into $n$ discrete states. At each state $i$, the probability of jumping in the neighborhood to the left or right is given, respectively, by $l_{i}$ and $r_{i}\left(l_{i}+r_{i}=1\right)$. The fractional Eq. (37) for $p_{i}(t)=\operatorname{Pr}\{X(t)=i\}$ can be rewritten as

$$
p_{i}^{\prime}(t)=\frac{r_{i-1} \mathcal{D}_{t}^{1-\mu_{i-1}} p_{i-1}(t)}{\tau_{i-1}^{\mu_{i-1}}}+\frac{l_{i+1} \mathcal{D}_{t}^{1-\mu_{i+1}} p_{i+1}(t)}{\tau_{i+1}^{\mu_{i+1}}}
$$

$$
-\frac{\mathcal{D}_{t}^{1-\mu_{i-1}} p_{i}(t)}{\tau_{i}^{\mu_{i}}}, \quad i=1, \ldots, n,
$$

subject to the conditions $l_{1}=r_{-1}=0, r_{1}=1$ and $l_{n}=1$, $r_{n}=l_{n+1}=0$. Note that the FFP Eq. (38) is just a continuous approximation of Eq. (41). Taking the Laplace transform of (41) and using $\sum_{i} \hat{p}_{i}(s)=\frac{1}{s}$, we obtain

$$
\begin{aligned}
s \hat{p}_{i}(s) & \left(1+\frac{r_{i-1}}{\left(s \tau_{i-1}\right)^{\mu_{i-1}}}+\frac{l_{i+1}}{\left(s \tau_{i+1}\right)^{\mu_{i+1}}}+\frac{1}{\left(s \tau_{i}\right)^{\mu_{i}}}\right) \\
= & \frac{r_{i-1}}{\left(s \tau_{i-1}\right)^{\mu_{i-1}}}\left(1-\sum_{j \neq i-1, i} s \hat{p}_{j}(s)\right) \\
& +\frac{l_{i+1}}{\left(s \tau_{i+1}\right)^{\mu_{i+1}}}\left(1-\sum_{j \neq i, i+1} s \hat{p}_{j}(s)\right)+p_{i}(0) .
\end{aligned}
$$

If one $\mu_{M}$ is smaller than the others $\left(\mu_{M}<\mu_{i} \forall i\right)$, one can find that $s \hat{p}_{i}(s) \rightarrow 0$ and $s \hat{p}_{M}(s) \rightarrow 1$ as $s \rightarrow 0$. It means that in the limit $t \rightarrow \infty$, we obtain

$$
p_{i}(t) \rightarrow 0, \quad p_{M}(t) \rightarrow 1 .
$$

This result in a continuous case can be rewritten as $p(x, t) \rightarrow$ $\delta\left(x-x_{\min }\right)$ as $\rightarrow \infty$, where $x_{\min }$ is the point on the interval $[0, L]$ at which $\mu(x)$ takes its minimum value. A similar result was obtained for a symmetrical random walk in Ref. [17] in the context of chemotaxis (anomalous aggregation). Note that Shushin [21] considered a two-state anomalous system with a different anomalous exponent $\mu$ and found that in the long-time limit the probability is located in the slower state (see also [12,22]).

\section{MONTE CARLO SIMULATIONS}

To validate our results, we run Monte Carlo simulations with the following procedure. Random numbers with uniform distribution, $u$ and $v$, are generated and then transformed into Mittag-Leffler distributed random numbers using the following inversion formula $t_{\mu}=-\tau \log (u)\left(\frac{\sin (\mu \pi)}{\tan (\mu \pi v)}-\cos (\mu \pi)\right)^{\frac{1}{\mu}}$ [24] (see for details [25]). We take $L=1$ and divide the interval $[0,1]$ into 100 subintervals. We use $r_{i}=1 / 2+5 a(1-$ $2 a i) / 2,1 \leqslant i \leqslant 100$ and $a=1 / 100$. This corresponds to

$$
r(x)=\frac{1}{2}+5 a\left(\frac{1}{2}-x\right),
$$

so the drift $v_{\mu}(x)=10(1-2 x) D_{\mu}$ and the potential,

$$
U(x)=\frac{5}{2}(1-2 x)^{2} .
$$

All the random walkers start in the same state $i=40$, their number $N=10^{4}, \tau_{i}=10^{-4}$ for all $i$, and the long-time limit is set at $T=10^{5}$.

First step is to compute the exact stationary PDF given by Eq. (40) and see how well our Monte Carlo simulations work. Figure 2 shows that the Monte Carlo simulations agree with the Gibbs-Boltzmann distribution.

The next step is to show that the Gibbs-Boltzmann distribution (40) is absolutely irrelevant as far as the longtime behavior of the nonuniform system is concerned. The anomalous exponent $\mu_{i}$ is assumed to be 0.5 for all states except one, $i=42$, for which $\mu_{42}=0.3$. One can see from Fig. 3 that in the long-time limit the probability is concentrated 




FIG. 4. The PDF $f(\mu)$ of random anomalous exponent $\mu$.

at state $i=42$. One can conclude that there is a complete breakdown in the predictions based on the FFP equation with the uniform anomalous exponent. If the system were structurally stable we would expect to see something more like Fig. 2 again. However, the outcome is completely dominated by the perturbation $\mu_{42}=0.3$. This result has a huge implication for modeling the anomalous subdiffusive transport of proteins, porous media, etc. In reality the environment in which anomalous transport takes place is never homogeneous.

Several attempts have been made to take into account the random distribution of the anomalous exponent (see, e.g., $[26,27])$. One can introduce $\operatorname{PDF} f(\mu)$ for a random $\mu$ and write down the distributed-order fractional FPE as

$$
\int_{0}^{1} \tau^{\mu-1} \frac{\partial^{\mu} p}{\partial t^{\mu}} f(\mu) d \mu=L_{F P} p .
$$

Let us show that if we generate the random field $\mu(x)$ along the space interval $[0,1]$, the asymptotic behavior of $p(x, t)$ will be quite different from that of the average fractional Eq. (46).

Figure 4 shows the PDF $f(\mu)$ which will be used to generate the discrete uncorrelated random field $\mu_{i}$. The probability is concentrated around the point 0.6 such that $\operatorname{Pr}\{0.5<\mu<0.7\}=0.98$. This distribution is chosen so that extreme values are highly unlikely to occur, with a purpose to show that the extreme low values dominate the long-time behavior. Figure 5 shows one sample of random field $\mu(x)$ on the interval $[0,1]$ which is subdivided into 100 subintervals $(1 \leqslant i \leqslant 100)$. Figure 5 shows clearly that the values of $\mu_{i}$ fluctuate around the mean. The value at $\mu_{82}=0.01245$ has

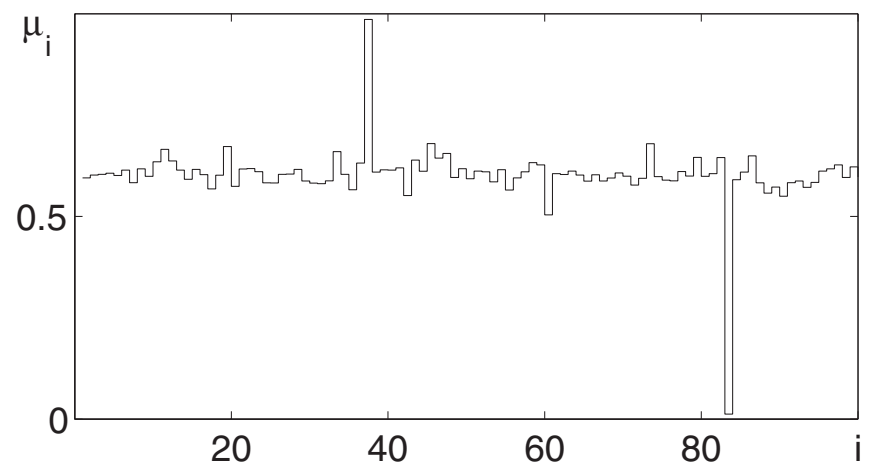

FIG. 5. One sample of the discrete random field $\mu_{i}$ along $i$ for $1 \leqslant i \leqslant 100$.

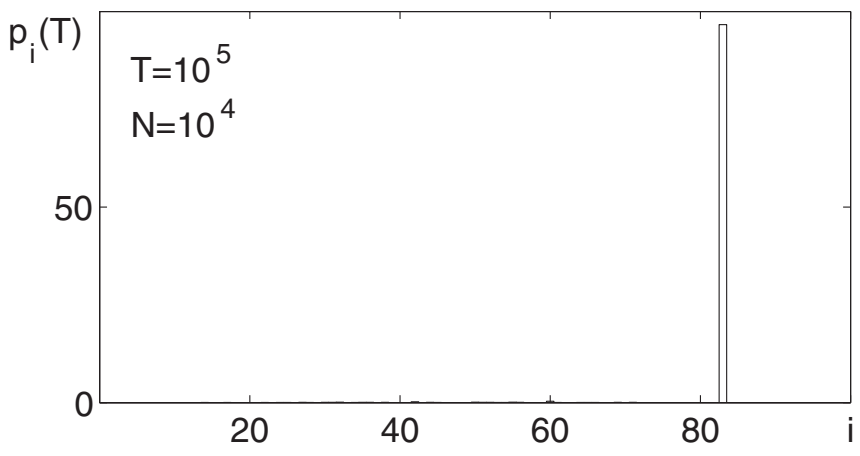

FIG. 6. Long-time limit of the system (41) when $\mu_{i}$ is the random field represented in Fig. 5.

a very small probability, since $\operatorname{Pr}\{\mu<0.02\}=2.5 \times 10^{-4}$. It is a very unlikely event, yet one can see from Fig. 6 the state $i=82$ completely dominates the long-time outcome of Eq. (41). This phenomenon can be interpreted as a "Black Swan." We use the term "Black Swan," to capture the idea proposed by Taleb [28] of the disproportionate role of rare events with extreme impact. Here the "Black Swan" is an outlier (small value of anomalous exponent) that completely dominates the long-time behavior of subdiffusive systems. This event has a very low probability of happening. However, when it does occur it has a high impact on the future evolution of the process.

The distribution of $p(x, t)$ is highly intermediate for large $t$, so the average behavior described by Eq. (46) can be very misleading. It has been found [26] that the distribution of the anomalous exponent in Eq. (46) leads to ultraslow kinetics, but the stationary distribution is still given by the Gibbs-Boltzmann distribution [23]. Our results show that random space variation of the anomalous exponent leads to completely different behavior in the long-time limit (see Fig. 6). It should be noted that anomalous diffusion is just an intermediate asymptotic. When time tends to infinity we expect a crossover from anomalous diffusion to normal diffusion, and then we will recover the Gibbs-Boltzmann distribution. The standard tool for studying a subdiffusion is a subordination technique [29] with a constant anomalous exponent. It would be interesting to apply a similar technique if possible to the nonhomogeneous case. It would be also interesting to take into account chemical reactions together with the nonuniform anomalous exponent [30].

\section{CONCLUSIONS}

We have demonstrated that when the anomalous exponent $\mu$ depends on the space variable $x$, the Gibbs-Boltzmann distribution is not a long-time limit of the fractional FokkerPlanck equation. Even very small variations of the exponent lead to a drastic change of $p(x, t)$ in the limit $t \rightarrow \infty$. We have derived the fractional master equation with the spacedependent anomalous exponent. We analyzed asymptotic behavior of the corresponding lattice model in a finite domain with $n$ states with different exponents. We have found that in this situation the probabilities $p_{i}(t)$ do not converge to the stationary distribution. To illustrate our ideas, we ran Monte Carlo simulations which show a complete breakdown 
in the predictions based on the FFP equation with the uniform anomalous exponent. Furthermore, we have shown that the idea of taking into account the randomness of the anomalous exponent $\mu$ by averaging the fractional equation with respect to the distribution $f(\mu)$ is not applicable to a nonhomogeneous finite domain. Monte Carlo simulations show that for every random realization of $\mu(x)$ the PDF $p(x, t)$ is highly intermediate, so the average behavior can be misleading. Although it is possible in theory to have a completely homogeneous environment, in which $\mu$ is uniform, it is not useful in any real application like chemotaxis [17] or morphogen gradient formation [31] because any nonhomogeneous variation destroys the predictions based on this model in the long-time limit. We have demonstrated that subdiffusive equations with the nonuniform random distribution of the anomalous exponent illustrate the "Black Swan" phenomenon [28], when an outlier (small value of anomalous exponent) completely dominates the long-time behavior of subdiffusive systems.
[1] R. Metzler and J. Klafter, Phys. Rep. 339, 1 (2000).

[2] R. Metzler and J. Klafter, J. Phys. A: Math. Gen. 37, R161 (2004).

[3] Anomalous Transport: Foundations and Applications, edited by R. Klages, G. Radons, and I. M. Sokolov (Wiley-VCH, Weinheim, 2008).

[4] V. Méndez, S. Fedotov, and W. Horsthemke, Reaction-Transport Systems (Springer, Berlin, 2010).

[5] M. J. Saxton, Biophys. J. 81, 2226 (2001).

[6] I. Golding and E. C. Cox, Phys. Rev. Lett. 96, 098102 (2006).

[7] F. Santamaria, S. Wils, E. De Schutter, and G. J. Augustine, Neuron 52, 635 (2006); S. Fedotov and V. Méndez, Phys. Rev. Lett. 101, 218102 (2008).

[8] C. W. Gardiner, Handbook of Stochastic Methods, 3rd ed. (Springer, Berlin, 2004).

[9] N. N. Leonenko, M. M. Meerschaert, and A. Sikorskii (to be published).

[10] D. S. Grebenkov, Phys. Rev. E 81, 021128 (2010).

[11] A. V. Chechkin, R. Gorenflo, and I. M. Sokolov, J. Phys. A: Math. Gen 38, L679 (2005).

[12] N. Korabel and E. Barkai, Phys. Rev. Lett. 104, 170603 (2010).

[13] B. A. Stickler and E. Schachinger, Phys. Rev. E 84, 021116 (2011).

[14] D. R. Cox and H. D. Miller, The Theory of Stochastic Processes (Methuen, London, 1965).

[15] M. O. Vlad and J. Ross, Phys. Rev. E 66, 061908 (2002).

[16] A. Yadav and W. Horsthemke, Phys. Rev. E 74, 066118 (2006).

[17] S. Fedotov, Phys. Rev. E 83, 021110 (2011).

[18] E. Scalas, R. Gorenflo, F. Mainardi, and M. Raberto, Fractals 11, 281 (2003).
[19] B. I. Henry, T. A. M. Langlands, and P. Straka, Phys. Rev. Lett. 105, 170602 (2010).

[20] E. Barkai, R. Metzler, and J. Klafter, Phys. Rev. E 61, 132 (2000).

[21] A. I. Shushin, Phys. Rev. E 64, 051108 (2001).

[22] D. Campos, S. Fedotov, and V. Méndez, Phys. Rev. E 77, 061130 (2008).

[23] B. Dybiec, I. M. Sokolov, and A. V. Chechkin, Comm. Nonlinear Sci. Numer. Simulat. 16, 4549 (2011).

[24] T. J. Kozubowski and S. T. Rachev, J. Computational Analysis and Applications 1, 177 (1999).

[25] D. Fulger, E. Scalas, and G. Germano, Phys. Rev. E 77, 021122 (2008).

[26] A. V. Chechkin, J. Klafter, and I. M. Sokolov, Europhys. Lett. 63, 326 (2003).

[27] M. M. Meerschaert, E. Nane, and P. Vellaisamy, J. Math. Anal. Appl. 379, 216 (2011).

[28] N. Taleb, The Black Swan: The Impact of the Highly Improbable (Random House, New York, 2007).

[29] M. M. Meerschaert, D. A. Benson, H. P. Scheffler, and B. Baeumer, Phys. Rev. E 65, 041103 (2002); M. Magdziarz, A. Weron, and K. Weron, ibid. 75, 016708 (2007); A. Weron, M. Magdziarz, and K. Weron, ibid. 77, 036704 (2008); V. Kolokoltsov, Theory Probab. Appl. 53, 594 (2009); R. Gorenflo and F. Mainardi, Eur. Phys. J. Special Topics 193, 119 (2011).

[30] A. Yadav and W. Horsthemke, Phys. Rev. E 74, 066118 (2006); Y. Nec and A. A. Nepomnyashchy, J. Phys. A: Math. Theor. 40, 14687 (2007); S. Fedotov and A. Iomin, Phys. Rev. Lett. 98, 118101 (2007); S. Fedotov, Phys. Rev. E 81, 011117 (2010).

[31] S. B. Yuste, E. Abad, and Katja Lindenberg, Phys. Rev. E 82, 061123 (2010). 